\title{
Systematic review of chewing simulators: Reality and reproducibility of in vitro studies
}

\author{
Sergio Soriano-Valero ${ }^{1}$, Juan-Luis Román-Rodriguez ${ }^{2}$, Rubén Agustín-Panadero ${ }^{2}$, Carlos Bellot-Arcís ${ }^{3}$, \\ Antonio Fons-Font ${ }^{2}$, Lucía Fernández-Estevan ${ }^{2}$ \\ ${ }^{1}$ Private Practice, Alicante, Spain \\ ${ }^{2}$ Prosthodontics and Occlusion Unit, Department of Stomatology, Faculty of Medicine and Dentistry, University of Valencia, \\ 46010 Valencia, Spain \\ ${ }^{3}$ Orthodontics Teaching Unit, Department of Stomatology, Faculty of Medicine and Dentistry, University of Valencia, 46010 \\ Valencia, Spain
}

Correspondence:

Faculty of Medicine and Dentistry

(Unit of Prosthodontics and Occlusion)

Universitat de València

C/ Gascó Oliag, 1. 46010 Valencia, Spain

ruben.agustin@uv.es

Soriano-Valero S, Román-Rodriguez JL, Agustín-Panadero R, Bellot-Arcís C, Fons-Font A, Fernández-Estevan L. Systematic review of chewing simulators: Reality and reproducibility of in vitro studies. J Clin Exp Dent.

Received: 06/05/2020 Accepted: 02/07/2020 2020;12(12):e1189-95.

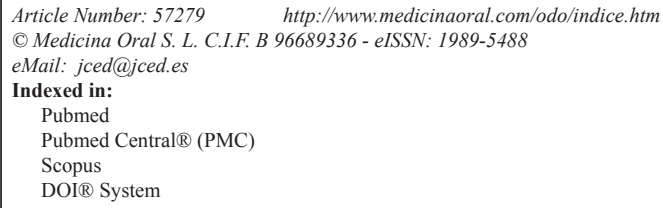

\begin{abstract}
Background: The aim of this systematic review was to analyze the types of human chewing simulator described in scientific literature.

Material and Methods: An electronic search was conducted in the databases PubMed, Embase and Scopus. The search strategy included 10 search terms: "in vitro"; "dental materials"; "shear strength"; "fatigue fracture"; "bite force"; "prosthetic materials"; "chewing simulator"; "chewing machine"; "simulated mastication"; and "dental wear simulator." Two researchers worked independently to assess the titles and abstracts of the articles. The quality of the in vitro trials selected was evaluated by means of the Consolidated Standards of Reporting Trials scale. Results: The electronic search identified 80 articles related to the topic of interest. After reading the full texts, ten works were selected. The articles focused mainly on the design of chewing simulators. Most of them were considered of moderate quality. Regarding the characteristics that an ideal chewing simulator should encompass, the devices described in articles varied greatly in terms of movement, periodontal ligament simulation, force sensors, and the materials tested.

Conclusions: No chewing simulator offers all the characteristics necessary to reproduce human masticatory movements and forces under the humidity and $\mathrm{pH}$ conditions of the oral cavity. A simulator that encompasses all these characteristics would make it possible to standardize trials involving simulated mastication.
\end{abstract}

Key words: In vitro, dental materials, dental wear simulator. 


\section{Introduction}

The mechanical properties of the materials used in dentistry must be thoroughly tested under laboratory conditions before they can enter clinical use. Trials of mechanical resistance (hardness, fracture resistance, elasticity modulus, etc.) must be complemented by wear and fatigue testing.

Wear means "to damage, erode, or destroy by friction or use," while fatigue means "weakness in metal or other materials caused by repeated variations of stress." In dentistry the usual terms used to describe these scenarios are aging and fatigue (1).

The need for information about the wear and fatigue characteristics of dental materials before they enter clinical use has led to the development of a range of devices intended to simulate mastication. These simulators are used before final load testing to provide information about a material's behavior during prolonged use. At the same time, many in vitro trials need to imitate, as far as possible, the physiological characteristics of human mastication, and the direction and force of jaw movements $(2,3)$.

The Federal Drug Administration (FDA) has established a series of directives for good laboratory practice. But to date, no single standardized system has been determined for measuring wear and aging, as is the case when measuring traction and fracture resistance. In the currently available chewing simulators, variability in terms of the control and regulation of forces impacts negatively on the reproducibility and variability of results, and the difficulty of extrapolating in vitro findings to the oral cavity (4).

The aim of the present systematic review was to analyze the types of chewing simulator described in scientific literature.

\section{Material and Methods}

This systematic review was conducted in accordance with Preferred Reporting Items for Systematic Reviews and Meta-Analyses (PRISMA) (5). It could not be registered in the PROSPERO database as it investigated in vitro studies.

The review's PICO (participants, intervention, comparison, outcome) question was: Which chewing simulator most resembles human mastication, in which $\mathrm{P}=$ chewing simulators; $\mathrm{I}=$ in vitro mastication; $\mathrm{C}=$ analysis; and $\mathrm{O}=$ human mastication.

The search identified articles that analyzed in vitro wear resulting from the action of chewing simulators. Inclusion criteria were: chewing device design was described, and test samples were described (teeth studied/antagonist teeth). These criteria were chosen to center the review on articles involving chewing simulation and oral conditions.

Firstly, an electronic search was conducted in the databases PubMed, Embase and Scopus. The search strategy included 10 search terms: "in vitro"; "dental materials"; "shear strength"; "fatigue fracture"; "bite force"; "prosthetic materials"; "chewing simulator"; "chewing machine"; "simulated mastication"; and "dental wear simulator". Boolean operators ("OR" and "AND”) were applied to link search terms to the research question (Table 1).

Two reviewers worked independently (L.F.-E. and S.S.-V.) to assess the titles and abstracts of the articles identified in the initial search. The following variables were recorded for each article: author and year of publication, device, study teeth/antagonist teeth, simulated mastication movement, force sensors.

To assess the in vitro studies included in analysis, their quality was evaluated by means of the Consolidated Standards of Reporting Trials (CONSORT) scale, mo-

Table 1: Search strategy used to locate studies in primary electronic databases.

\begin{tabular}{|c|c|}
\hline Databases & Key search terms \\
\hline Pubmed & $\begin{array}{c}\text { (((()in vitro }[\mathrm{MeSH} \text { Terms] }) \text { OR prosthetic materials) OR dental materials }[\mathrm{MeSH} \\
\text { Terms])) AND ((((chewing simulator) OR chewing machine) OR simulated mastica- } \\
\text { tion) OR dental wear simulator)) AND (((shear strength }[\mathrm{MeSH} \text { Terms]) OR fatigue } \\
\text { fracture }[\mathrm{MeSH} \text { Terms] }) \text { OR bite force }[\mathrm{MeSH} \text { Terms] })\end{array}$ \\
\hline Embase & $\begin{array}{l}\text { ((in AND vitro OR prosthetic) AND ('materials'/exp OR materials) OR 'dental } \\
\text { material'/exp OR 'dental material') AND (((('chewing'/exp OR chewing) AND } \\
\text { ('simulator'/exp OR simulator) OR 'chewing'/exp OR chewing) AND ('machine'/exp } \\
\text { OR machine) OR simulated) AND ('mastication'/exp OR mastication) OR 'dental'/ } \\
\text { exp OR dental) AND ('wear'/exp OR wear) AND ('simulator'/exp OR simulator) } \\
\text { AND ((shearAND ('strength'/exp OR strength) OR 'fatigue'/exp OR fatigue) AND } \\
\text { ('fracture'/exp OR fracture) OR 'bite'/exp OR bite) AND ('force'/exp OR force) }\end{array}$ \\
\hline Scopus & $\begin{array}{l}\text { ((((in AND vitro [mesh AND terms]) OR prosthetic AND materials) OR dental A } \\
\text { ND materials [mesh AND terms])) AND ((((chewing AND simulator) OR chewing } \\
\text { AND machine) OR simulated AND mastication) OR dental AND wear AND simu- } \\
\text { lator)) AND (((shear AND strength [mesh AND terms]) OR fatigue AND fracture } \\
\text { [mesh AND terms]) OR bite AND force [mesh AND terms]) }\end{array}$ \\
\hline
\end{tabular}


dified to assess the quality of in vitro trials of dental materials (6). This modified CONSORT scale consists of 15 items that assess the quality of studies in terms of the abstract, introduction, method, results, discussion and other information of interest such as financial support received or access to a description of the test protocol employed. Items included in an article are marked with an asterisk; when a box remains blank this means that the item has not been mentioned in the text.

\section{Results}

The electronic search identified 80 references related to the research topic, 73 in Pubmed, 3 in Embase and 4 in Scopus. Fifty-seven articles were excluded on the basis of the title, and a further nine after reading the abstracts. After reading the full texts, another four articles were excluded as they failed to provide information relevant to the review (Fig. 1). Finally, 10 articles were selected for review (Table 2), all of which focused to greater or lesser extent on chewing simulator design.
The ten articles selected for review were in vitro experimental studies. Their quality was evaluated by means of the CONSORT scale. Most of them were considered of moderate quality, as all fulfilled around 8 items out of the total of 15. Table 3 shows the results of the CONSORT scale. All works included a complete abstract (item 1). They also included an introduction that described the study's scientific antecedents and purpose (item 2a), objectives, and/or hypothesis (item $2 b$ ). Regarding the methods section, all explained the intervention carried out on each material evaluated (item 3 ), and the data recorded in the results, including a description of the test procedure and moment when each material was tested (item 4). The method used to generate a random allocation sequence location and the mechanism used to implement the random allocation sequence (items 6 and 7) were only found in five articles. The statistical methods applied were described in seven works (item 10). For each primary and secondary outcome, results for each group, and the estimated size of the effect and its preci-
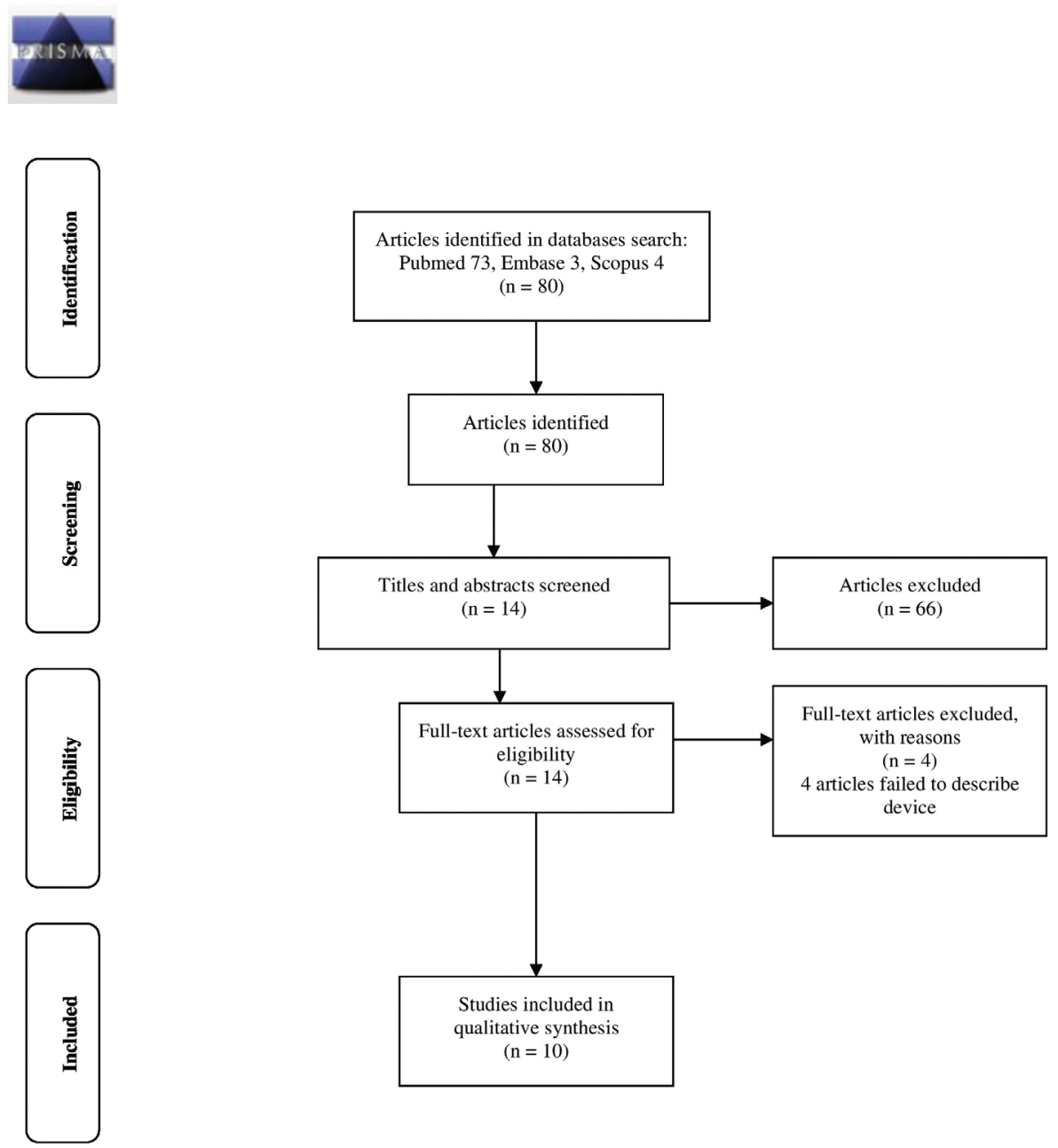

Fig. 1: PRISMA 2009 Flow Diagram. 
Table 2: Variables analyzed in systematic review.

\begin{tabular}{|c|c|c|c|c|}
\hline $\begin{array}{c}\text { Author, year of } \\
\text { publication }\end{array}$ & Device & Teeth/antagonist studied & Movement & Force sensor \\
\hline Jensen, 2007 & $\begin{array}{l}\text { Willytec (fracture } \\
\text { resistance/wear) }\end{array}$ & $\begin{array}{l}\text { Natural tooth (apex covered in wax)/ } \\
\text { Natural tooth on acrylic resin base }\end{array}$ & $\begin{array}{l}\text { Vertical and lateral } \\
\text { movement }\end{array}$ & No \\
\hline Alemzadeh, 2007 & $\begin{array}{l}\text { Dento-Munch } \\
\text { (wear) }\end{array}$ & Artificial teeth/artificial teeth & Compression force & Yes \\
\hline Conserva, 2009 & $\begin{array}{l}\text { Masticatory robot } \\
\text { (fatigue) }\end{array}$ & $\begin{array}{l}\text { Stylus (simulating abutment-implant) } \\
\text { steel support + cobalt-chrome } \\
\text { maxillary arch (optional) }\end{array}$ & $\begin{array}{l}\text { Movement in three } \\
\text { dimensions }\end{array}$ & Yes \\
\hline Michalakis, 2009 & Instron (fatigue) & $\begin{array}{c}\text { Cobalt-chrome tooth (central incisor) } \\
\text { set in acrylic resin /stainless steel } \\
\text { stylus }\end{array}$ & Compression force & No \\
\hline Rues, 2011 & Willytec (wear) & $\begin{array}{l}\text { Enamel ball and restoration materials/ } \\
\text { aluminum ball }\end{array}$ & Compression force & Yes \\
\hline Heintze, 2011 & $\begin{array}{l}\text { Willytec (fracture } \\
\text { resistance/wear) }\end{array}$ & $\begin{array}{l}\text { First lower molar: PMMA* (CAD/ } \\
\text { CAM) }{ }^{\dagger / \text { stylus }}\end{array}$ & Eccentric & Yes \\
\hline Raabe, 2012 & $\begin{array}{l}\text { Robot dental wear } \\
\text { simulator (wear) }\end{array}$ & Tooth or arch/steatite ball & $\begin{array}{l}\text { Movement in three } \\
\text { dimensions }\end{array}$ & Yes \\
\hline Ruben, 2014 & Rub \& Roll (wear) & $\begin{array}{l}\text { Natural tooth, restoration material on } \\
\text { PMMA/rod }\end{array}$ & Lateral movement & Yes \\
\hline Shahin, 2014 & $\begin{array}{l}\text { Willytec (fracture } \\
\text { resistance/wear) }\end{array}$ & $\begin{array}{l}\text { Natural teeth on copper cylinder/ } \\
\text { steatite ball }\end{array}$ & Lateral movement & No \\
\hline Singhatanadgit, 2016 & $\begin{array}{c}\text { Mastication } \\
\text { simulator (fatigue) }\end{array}$ & $\begin{array}{l}\text { Natural premolars/tungsten carbide } \\
\text { stylus }\end{array}$ & $\begin{array}{l}\text { Uni- or bilateral } \\
\text { pathway }\end{array}$ & Yes \\
\hline
\end{tabular}

* PMMA, Polymethyl methacrylate. $\dagger$ CAD/CAM, Computer-aided design/computer-aided manufacturing.

Table 3: Quality of articles assessed with modified CONSORT scale for in vitro studies. Criteria: 1) Structured abstract; 2a) Scientific antecedents; 2b) Objectives and/or hypothesis; 3) Intervention; 4) Way and moment when outcomes were evaluated; 5) Sample size determination; 6) Method used to generate a random allocation sequence; 7) Mechanism used to generate a random allocation sequence; 8) Who generated random allocation sequence; 9) Who was blinded to random allocation and how; 10) Statistical methods for comparing outcomes; 11) Precision of results obtained; 12) Study limitations; 13) Sources of finance; 14) Access provided to study protocol.

\begin{tabular}{|l|c|c|c|c|c|c|c|c|c|c|c|c|c|c|c|}
\hline Studies & $\mathbf{1}$ & $\mathbf{2 a}$ & $\mathbf{2 b}$ & $\mathbf{3}$ & $\mathbf{4}$ & $\mathbf{5}$ & $\mathbf{6}$ & $\mathbf{7}$ & $\mathbf{8}$ & $\mathbf{9}$ & $\mathbf{1 0}$ & $\mathbf{1 1}$ & $\mathbf{1 2}$ & $\mathbf{1 3}$ & $\mathbf{1 4}$ \\
\hline Jensen, 2007 & $*$ & $*$ & $*$ & $*$ & $*$ & & $*$ & $*$ & & & $*$ & & $*$ & $*$ & \\
\hline Alemzadeh, 2007 & $*$ & $*$ & $*$ & $*$ & $*$ & & & & & & & & $*$ & & \\
\hline Conserva, 2009 & $*$ & $*$ & $*$ & $*$ & $*$ & & $*$ & $*$ & & & $*$ & $*$ & $*$ & $*$ & \\
\hline Michalakis, 2009 & $*$ & $*$ & $*$ & $*$ & $*$ & & & & & & $*$ & & $*$ & & \\
\hline Heintze, 2011 & $*$ & $*$ & $*$ & $*$ & $*$ & & & & & & $*$ & $*$ & $*$ & & \\
\hline Rues, 2011 & $*$ & $*$ & $*$ & $*$ & $*$ & & & & & & $*$ & & $*$ & & \\
\hline Raabe, 2012 & $*$ & $*$ & $*$ & $*$ & $*$ & & & & & & & & $*$ & $*$ & \\
\hline Ruben, 2014 & $*$ & $*$ & $*$ & $*$ & $*$ & & $*$ & $*$ & & & $*$ & & $*$ & & \\
\hline Shahin, 2014 & $*$ & $*$ & $*$ & $*$ & $*$ & & $*$ & $*$ & & & $*$ & & $*$ & $*$ & $*$ \\
\hline $\begin{array}{l}\text { Singhatanadgit, } \\
\text { 2016 }\end{array}$ & $*$ & $*$ & $*$ & $*$ & $*$ & & $*$ & $*$ & & & & & $*$ & $*$ \\
\hline
\end{tabular}

sion (item 11) were only reported in two articles. All the works suffered trial limitations (item 12). Five articles reported sources of funding. None of the studies explained how the sample size was determined (item 5), who generated the random allocation sequence, who enrolled teeth, who assigned teeth to intervention (item 8), who was blinded and how (item 9), or where the full trial protocol could be accessed (item 14).

The chewing simulators described were used for fatigue testing, and to measure resistance to fracture and wear. 
Jensen et al. (7), Heintze et al. (8), Rues et al. (4), and Shahin et al. (9), employed the Willytec chewing simulator (SD Mechatronic GmbH, Feldkirchen-Westerham, Germany). It has two motor driven axes to simulate different motion patterns, which are controlled by software that can program all the paths of the masticatory cycle. This device can be used to analyze the wear/fracture resistance of materials used in implants, crowns, bridges, composite restorations, jaw models; the antagonist can be a natural tooth, a carbide or steatite sphere, etc. The simulator is equipped with eight test chambers that can be used simultaneously (although there are smaller models with two or four chambers), each with an individual bar and weight; all the bars are linked by a transversal bar driven by a motor, which ensures that the test conditions are identical in each chamber. It is possible to modify the active axis's load as well as its sliding motion. The chambers can be filled with water or left unfilled.

The article by Michalakis et al. (10) employed a universal fatigue test machine (Instron Corp, Norwood, Mass, USA).

The masticatory robot described by Conserva et al. $(2,11)$ consists of two parts, the first being the robot and its control system, while the second records data. The control system is an industrial computer that gives orders to the robot's moving parts, a Stewart platform, controlling the movements it makes assisted by feedback signals. The Stewart platform is a parallel mechanism formed by an (upper) mobile platform (which simulates the mandible), connected to a fixed base by six identical kinetic jacks, equally spaced, symmetrically arranged to form two equilateral triangles over a fixed base. When the lengths of the jacks are varied by three linear force transmitters, it is possible to change the tilt of the platform through six degrees of freedom (three degrees of freedom for rotation, and three for tilting) replicating the force and motion of functional mastication. Each leg is made up of two steel cylinders each joined to the platform and the base by ball joints at each end. The robot's motion is determined by the platform position in $\mathrm{x}, \mathrm{y}$, and $\mathrm{z}$ positions and the platform's orientation defined as angles to the axes. The $\mathrm{x}, \mathrm{y}, \mathrm{z}$ axes represent the latero-lateral, anteroposterior and vertical axes, respectively. The antagonist is a steel plate, with the option of adding a cobalt-chrome maxilla. This simulator is used to analyze material fatigue.

Singhatanadgit et al. (3) developed a simulator designed to generate both unidirectional and bidirectional movements. It consists of two main parts: the upper part replicates the maxilla and the lower simulates the movement of the mandible. It uses a four-bar linkage mechanism to replicate mandibular motion and is used to analyze material fatigue.

Raabe et al. (12) employed a simulator consisting of a platform that replicates the static maxilla or any anta- gonist. The (lower) mobile portion impacts on the static part by means of six propelling arms which, in combination, simulate mandibular movements of variable force. The lower platform is equipped with a resilient system that imitates periodontal ligament. It also has a wet chamber that can be used with water, artificial saliva or other liquids.

Ruben et al. (13) proposed a machine - they called it "Rub \& Roll" - for wear-testing materials placed in a gyrating cylinder that generates a rubbing action between a material inserted in the cylinder and a static surface, which can be of any type of material.

Alemzadeh et al. (14) employed a device they called "Dento-Munch." This consists of a fixed base (lower plate) that does not move and platform (upper plate) which is set parallel to the lower plate and moves with six degrees of freedom varying the length of one or more of the force transmitters; this was used to study wear to materials.

Regarding the materials used both for test teeth/structures and antagonist teeth/structures, of the 10 articles reviewed, three used natural teeth as the test object $(3,7,9)$, one used a simulated implant-abutment set-up (11), one employed cobalt-chrome teeth to which metal-ceramic crowns were cemented (10), one used standardized (CAD/CAM) PMMA mandibular molars (8), one involved different sample types (enamel or dentin discs, or natural molar-shaped samples, teeth with or without roots, restoration materials, etc.) (13) one used discs of enamel and of restoration materials (4), another used artificial teeth consisting of a resin crown retained on a ceramic root by universal resin cement (14), and one used universal resin composite as a material for reproducing dental specimens (12).

As for the antagonist teeth used in the papers reviewed, only one article used natural teeth set in an acrylic base (14). The other works used steel, tungsten carbide, aluminum or steatite as antagonist materials: one article used a steel element with the option of adding a cobalt-chrome maxillary arch (11), two used a stainless steel stylus $(8,10)$, one work used a PVC-coated stainless steel stylus (13), one used a tungsten carbide stylus (3), one used an aluminum sphere (4), and two a steatite ball $(9,12)$.

Various materials were used to simulate the behavior of periodontal ligament, aiming to imitate its resilience in force transmission. One study employed wax to replicate periodontal ligament (7), another silicon (9), while two used rubber $(13,14)$. A load cell was used in one study, placed below the static antagonist to imitate the feedback from periodontal proprioceptors (12). In the study by Michalakis et al. (10), cobalt-chrome teeth were set in self-curing acrylic resin blocks with the block surface placed $2 \mathrm{~mm}$ below the crown's margin. This design simulated the clinical conditions of a healthy periodon- 
tium. In the four remaining articles, no simulation of periodontal ligament was mentioned $(3,4,8,11)$.

In addition to the chewing simulator devices and the samples tested in them, it is important to analyze the movements made by the antagonist structures. In three studies, the devices generated a simulation of masticatory movement and masticatory forces in three dimensions $(3,11,12)$. In another three articles, the chewing simulators applied vertical compression forces exclusively $(4,10,14)$. Another simulator applied eccentric or lateral forces (13). In the three remaining studies, the simulators employed vertical and lateral movements but not three-dimensional motion (7-9).

In addition to the movements produced by the chewing simulator devices, the present review also analyzed the use (or absence) of load sensors for measuring the forces exerted. Seven articles stated the force applied. Conserva $e t$ al. (11) used a base equipped with a sensor fixed to the robot's mobile platform (mandible), which recorded the degree of force transmitted through the axes (x, y, and z). Heintze et al. (8) employed a 3D piezoelectric force sensor during dynamic loading; this was fixed to a special support at the lower end of the vertical load bar. Ruben et al. (13) used a single point load cell on the outside of the container equipped with a measuring sensor. Singhatanadgit et al. (3) employed a miniature load cell between the tungsten carbide stylus and the base of the mobile weight. Rues et al. (4) considered that, if there was only one force sensor available, then the eight chambers should be measured separately. Alemzadeh et al. (14) used extensiometric strain gauge force transducers on the mandible below the second molars to measure axial bite forces applied to posterior teeth. Raabe et al. (12) placed a load cell below the static antagonist. The three remaining works made no mention of having employed any force sensor $(7,9,10)$.

\section{Discussion}

In recent years, various chewing simulators have attempted to reproduce the oral environment in order to test dental materials under conditions as close as possible to in vivo conditions (15).

On the basis of the present review findings, the ideal chewing simulator should have specimen positioning devices that simulate human jaws. Among the articles reviewed, only one study placed natural teeth against the same type of antagonist, although this consisted of single teeth rather than complete arches (7). The literature does not describe any device that uses complete upper and lower jaws with natural teeth for wear testing.

The natural teeth used in these devices must be set on structures with similar characteristics to natural supporting structures (periodontal ligament and alveolar bone), made from materials that exhibit resilient rather than rigid behavior. In this context, silicon and acrylic resin have been used to imitate periodontal ligament and alveolar bone, respectively (16).

The simulator must be able to generate movements that imitate human mastication as far as a machine is able to. In mastication, the mandible moves in vertical and horizontal direction, making a bidirectional motion $(3,17)$. However, most of the devices fail to reproduce the complex movements of mastication in all three dimensions (2). The ideal simulator should be able to make opening and closing movements, as well as eccentric (lateral and protrusive) movements in order to analyze wear to materials subjected to the vertical and horizontal loads that occur in mastication motion. Although an ideal simulator does not yet exist, many of the simulators described above are able to generate motion that is not limited to compression alone (3,7-9,11-13).

If wear testing is to be standardized and obtain comparable results, it is essential for the magnitude of forces generated by chewing simulators to be controlled to create specific test conditions, and for these to be identical for each specimen tested. For this reason, the simulator must be equipped with load sensors to record the loads applied, as in some of the studies reviewed $(3,4,8,11$ 14). It is important to know the exact load applied to each dental group, as this will differ from group to group. To simulate conditions in the oral medium, some studies have attended to environmental factors such as humidity/wetness, temperature and $\mathrm{pH}$, which can also influence the mechanical properties and behavior of dental materials in the mouth. The body acts as a heat pump that maintains the temperature in the oral cavity at $37^{\circ} \mathrm{C}$. Teeth and any restoration material are continuously bathed in saliva with a $\mathrm{pH}$ of around 7. Humidity in the cavity is $100 \%$. However, the introduction of different foods in the mouth can alter environmental conditions to a large degree, causing fluctuations in $\mathrm{pH}$ and temperature. An artificial oral environment should be able to reproduce normal conditions in the oral cavity, and to manage the temperature and $\mathrm{pH}$ fluctuations that may occur. In vitro studies may use human saliva or de-ionized water, among other liquids, as a lubricant or even as an abrasive/erosive medium $(3,12,13,15,18)$.

There are few bibliographic references regarding the design of models for use in chewing simulators for trials involving simulated mastication. Several parameters have not been sufficiently investigated, including the curve of Spee and the curve of Wilson. Alemzadeh et al. (14) analyzed the curve of Spee digitally. Another relevant parameter is the angle of the occlusal plane at which maxillary models are set in the simulator; no research has contemplated this parameter in terms of replicating masticatory movements. It is necessary to standardize the values of these parameters in order to analyze wear to dental materials in models with identical characteristics. 
The present systematic review suffered certain limitations. Despite conducting a search in the databases, only a small number of relevant articles were identified, which might be explained by the difficulties involved in carrying out the type of in vitro study that was the subject of the review. The search did not include the Cochrane database as it does not admit in vitro research. The quality of most of the studies was moderate as it is hard for this type of in vitro research to meet the criteria that would deem it of high quality.

\section{Conclusions}

The present systematic review did not identify any chewing simulator that includes all the characteristics that would make it possible for complete upper and lower dental arches to reproduce the movements of human mastication under the actual humidity and $\mathrm{pH}$ conditions in the oral cavity. There is need for a simulator that meets all these requirements in order to standardize future trials involving simulated mastication.

\section{References}

1. Özcan M, Jonasch M. Effect of Cyclic Fatigue Tests on Aging and Their Translational Implications for Survival of All-Ceramic Tooth-Borne Single Crowns and Fixed Dental Prostheses. J Prosthodont. 2018;27:364-375.

2. Conserva E, Menini M, Tealdo T, Bevilacqua M, Pera F, Ravera G, et al. Robotic chewing simulator for dental materials testing on a sensor-equipped implant setup. Int J Prosthodont. 2008;21:501-8.

3. Singhatanadgit W, Junkaew P, Singhatanadgid P. Effect of bidirectional loading on contact and force characteristics under a newly developed masticatory simulator with a dual-direction loading system. Dent Mater J. 2016;35:952-961.

4. Rues S, Huber G, Rammelsberg P, Stober T. Effect of impact velocity and specimen stiffness on contact forces in a weight-controlled chewing simulator. Dent Mater. 2011;27:1267-72.

5. Moher D, Liberati A, Tetzlaff J, Altman DG. Preferred Reporting Items for Systematic Reviews and Meta-Analyses: The PRISMA Statement. PLoS Med. 2009;6:1000097.

6. Faggion CMJr. Guidelines for reporting pre-clinical in vitro studies on dental materials. J Evid Based Dent Pract. 2012;12:182-9.

7. Jensen AL, Abbott PV. Experimental model: dye penetration of extensive interim restorations used during endodontic treatment while under load in a multiple axis chewing simulator. J Endod. 2007;33:1243-6.

8. Heintze SD, Albrecht T, Cavalleri A, Steiner M. A new method to test the fracture probability of all-ceramic crowns with a dual-axis chewing simulator. Dent Mater. 2011;27:10-9.

9. Shahin R, Tannous F, Kern M. Inlay-retained cantilever fixed dental prostheses to substitute a single premolar: impact of zirconia framework design after dynamic loading. Eur J Oral Sci. 2014;122:310-6. 10. Michalakis KX, Stratos A, Hirayama H, Kang K, Touloumi F, Oishi Y. Fracture resistance of metal ceramic restorations with two different margin designs after exposure to masticatory simulation. $\mathrm{J}$ Prosthet Dent. 2009;102:172-8.

11. Conserva E, Menini M, Tealdo T, Bevilacqua M, Ravera G, Pera $\mathrm{F}$, et al. The use of a masticatory robot to analyze the shock absorption capacity of different restorative materials for prosthetic implants: a preliminary report. Int J Prosthodont. 2009;22:53-5.

12. Raabe D, Harrison A, Ireland A, Alemzadeh K, Sandy J, Dogramadzi S, et al. Improved single- and multi-contact life-time testing of dental restorative materials using key characteristics of the human masticatory system and a force/position-controlled robotic dental wear simulator. Bioinspir Biomim. 2012;7:016002.
13. Ruben JL, Roeters FJM, Montagner AF, Huysmans MCDNJM. A multifunctional device to simulate oral ageing: the "Rub\&Roll". J Mech Behav Biomed Mater. 2014;30:75-82.

14. Alemzadeh K, Raabe D. Prototyping artificial jaws for the Bristol Dento-Munch Robo-Simulator. 'A parallel robot to test dental components and materials'. Conf Proc IEEE Eng Med Biol Soc. 2007;2007:1453-6.

15. Abouelleil H, Jeannin C, Sadat A, Grosgogeat B. Development of a chewing simulator for testing dental materials: a pilot study. Br J Appl Sci Technol. 2015;5:1-8.

16. Aksel H, Askerbeyli-Örs S, Deniz-Sungur D. Vertical root fracture resistance of simulated immature permanent teeth filled with MTA using different vehicles. J Clin Exp Dent. 2017;9:178-181.

17. Rosentritt M, Schumann F, Krifka S, Preis V. Influence of zirconia and lithium disilicate tooth- or implant-supported crowns on wear of antagonistic and adjacent teeth. J Adv Prosthodont. 2020;12:1-8.

18. DeLong R, Douglas WH. An artificial oral environment for testing dental materials. IEEE Trans Biomed Eng. 1991;38:339-45.

\section{Conflict of interest}

Non declared. 